\title{
Research on Flashover Process of Uniform and Un-uniform Contaminated Insulator String
}

\author{
Yawei $\mathrm{Li}^{1, \text { a }}$, Xinghai Zhang ${ }^{1, \mathrm{~b}}$, Longchen Liu ${ }^{1, \mathrm{c}}$, Zongxi Zhang ${ }^{1, \mathrm{~d}}$, \\ Xiaolei Yang ${ }^{2, e}$ and Huan Bai ${ }^{1, f}$ \\ ${ }^{1}$ Electric Power Research Institute of State Grid Sichuan Electric Power Company, Chengdu \\ 610072, China. \\ ${ }^{2}$ State Grid Chengdu Power Supply Company, Chengdu 610041, China.

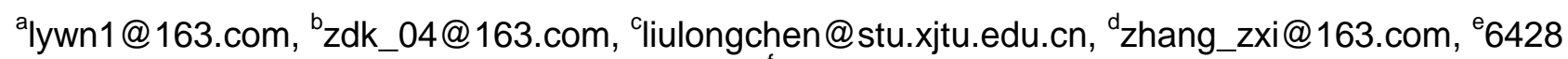 \\ 94834@qq.com, ‘371313060@qq.com
}

Keywords: Flashover, insulator, leakage current, partial arc.

\begin{abstract}
In order to clarify the differences of partial arc propagation process and flashover process between uniform and un-uniform contaminated insulator strings, experiments of flashover characteristics on uniform and un-uniform contaminated insulator strings have been made in this paper. The result indicates that the partial arc initial voltage decrease with increasing ESDD in uniform contamination distribution situation, relatively, in situation of un-uniform contamination distribution, the initial voltage of partial arc not only relevant to ESDD of the whole insulator string, but also the distribution of pollution layer. In the context uniform contamination distribution, the partial arcs develop as progressive-style; and in the case of un-uniform contamination distribution, the propagating mode of partial arcs is progressive-style and leap-style alternating. Assume that ESDD value of the whole strings constant, the number and lightness of partial arcs would decrease with the reduction of ratio between upper and lower surface ESDD values, and Arc-over occurrence probability as well. High amplitude pulses of leakage circuit exist in the course of flashover duration.
\end{abstract}

\section{Introduction}

The process of flashing involves ionization on the dirty surface concerning the factors of electric, heat and chemicals, thermal dynamic equilibrium process of local electric arc occurrence and development.

Because of the environmental pollution, polluted flashover accidents became more and more frequent since 1990's in China. According to statistics, the energy loss caused by flashover in 1986 1990 is 2.98 times of 10 years later, the flashover tripping operation probability of $110 \sim 500 \mathrm{kV}$ transmission lines sticks at a high level, and that of EHV transmission lines is much higher. Therefore, pollution flashover accidents become the main threaten of electric power system safety operation, and the impact of complicated environment condition over dielectric property of HV, EHV and UHV outdoor external insulation, is the most important technology problem.

In 1958, Obenhaus made a flashover model which is arc consists of residual layer resistance[1]. From then on, researchers around the world have done a lot of works to solve the flashover problem; many models based on Obenhaus' model have been brought forward, these researches play an important role in pollution flashover preventing. Nevertheless, the pollution flashover accidents are far from disappearing from the power system up to mow. As China's rapid economic development, atmospheric environment deteriorates gradually, serious acid rain increases, pollution flashover accidents become more frequent.

At home and abroad, many researches have been did in order to ravel the pollution flashover mechanism, characteristics, and preventing measures[2][3][4], but because of complicated influence factors, as the contamination distribution and shape, the physical process of pollution flashover 
discharge is not very clear so far, consequently, it is difficult to finding out an effective method to prevent the contaminated flashover once for all[5].

Therefore, it is necessary to analysis the partial arc propagation and flashover processes of insulator strings with different contamination distribution, each tested insulator string is composed of three XWP2-160 insulators. The differences of partial arc propagation and flashover processes at different pollution layer distribution would be revealed in this paper, and the precise description of partial arc and flashover would also be provided too. Based on these observation, the mechanism of partial arc propagation and flashover would be more approachable, it make a more accurate withstand voltage calculation model possible.

\section{Experimental Apparatus and Method Introduction}

\subsection{Experimental Apparatus.}

Experimental apparatus is shown in Fig.1, in which $\mathrm{T} 0$ is booster; $\mathrm{T}_{1}$ is testing transformer, rated voltage is $150 \mathrm{kV}$, rated current is $4 \mathrm{~A}$, short-circuit impedance is $5.47 \%$; $\mathrm{R} 0$ is protective resistance, $\mathrm{B}$ is wall bushing, $\mathrm{K}$ is artificial climate chamber, the diameter is $4 \mathrm{~m}$, height is $4.5 \mathrm{~m}$, climate chamber is metal, and reliably grounded, the $2.5 \mathrm{~m}$ height nozzles distribute on the wall, they are used for providing cold fog, the conductivity of tap water is no more than $500 \mu \mathrm{S} / \mathrm{cm}$, two fans are set up in both sides of the climate chamber to ensure that all parts of insulator strings can be saturated moist; D is resistance divider, the voltage ratio is $10000: 1$; $\mathrm{Y}$ is Rogowski coil, bandwidth is $1 \mathrm{~Hz} \sim 20 \mathrm{MHz}$, voltage ratio is 1:0.1; C is high-speed camera, shooting speed can up to 8000 pictures per second, it is used as recording arc development process; DPO is digital storage oscilloscope, be used as recording the voltage and current waveforms during the flashover experiment; I is tested insulator string, the type of insulators is $\mathrm{XWP}_{2}-160$.

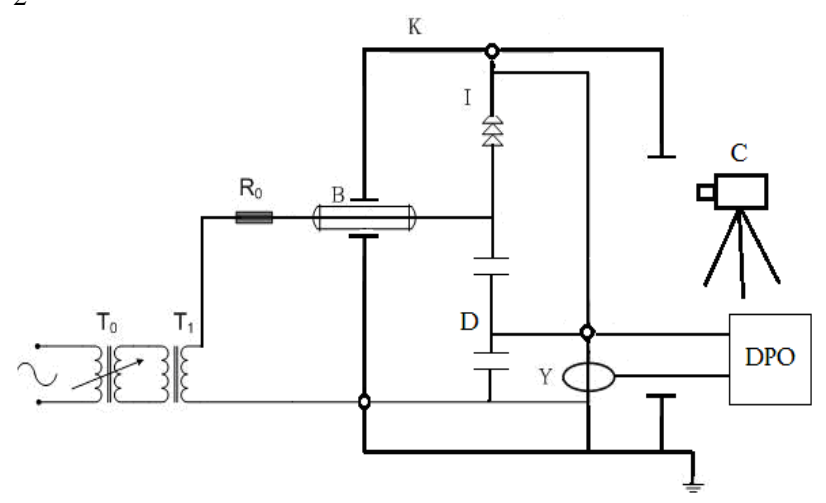

Fig 1. Schematic diagram of experimental apparatus

\subsection{Experimental methods.}

Quantitative brushing method is adopted in this paper. NSDD value is kept invariable as $1 \mathrm{mg} / \mathrm{cm}^{2}$, and ESDD value is respectively as $0.01,0.02,0.05,0.1,0.2\left(\mathrm{mg} / \mathrm{cm}^{2}\right)$ when uniform contaminated flashover tests are in progress.

For simulating the un-uniform pollution layer distribution, NSDD and ESDD value of upper surface and lower surface are proportional respectively as 1:2, 1:5, 1:8, 1:10, and the total NSDD of the whole insulator is $1 \mathrm{mg} / \mathrm{cm}^{2}$, total ESDD of the whole insulator is respectively as $0.05,0.1$, or 0.2 $\mathrm{mg} / \mathrm{cm}^{2}$. Put the cap upward, the upper surface of insulator is defined as the upper surface of both upper and lower skirts, and the rest is lower surface in this thesis. The upper and lower surface should be brushed separately after the weights of salt and kieselgur are calculated according to ESDD, NSDD and the area value of each region. After brushing, the insulator should be put in shady and dry place for about $6 \sim 8 \mathrm{~h}$.

After that, the insulator string is hang in the center of artificial climate chamber, open the spray switch, open the two fans in order to well-distribute the cold fog in the chamber. Up to droplets occur on the sheds of insulators, the pollution layer is completely moist. This process will spend 10min, and 
then shut the fans, open the door of chamber, make the high-speed camera ready, adjust the shooting speed to 1000 picture per second.

Uniform speed energization is employed in the flashover experiment. There is no restraint about the energizing speed when the acting voltage below $40 \%$ of the flashover voltage, after that, the set-up rate within 10\% 20\% of flashover voltage per second till completely flashover present at last.

Each insulator string flashover only one time in this experiment, the average flashover voltage value of 3 strings at the same contamination distribution is the flashover voltage of this condition. If the flashover voltage value changes out of $15 \%$ average voltage, replace a same insulator sting and repeat the test. Record the partial arc ignition, propagation and flashover processes by high speed camera, the voltage and current waveforms are recorded by digital storage oscilloscope in energizing duration.

\section{Analysis of Experimental Results}

\subsection{Analysis of Partial Arc Propagation Process.}

When contamination layer is wet completely, some moisture is absorbed by diatomaceous earth, the remain water dissolve $\mathrm{NaCl}$ to bring about electrolytic conductance, and lead to the increasing of surface leakage current, which thermal effect bring about dry bands.

Placing voltage on a single $\mathrm{XWP}_{2}-160$ Insulator, when the corona point appears, keeping voltage stable, and observing where the dry bands appear. Experimental results show that dry bands always appear near the pin at first, and then the cap, the region between the roots of two umbrella skirts. This is because the maximum leakage current density of the pin, and the current thermal effect is of the most significant, so initial dry bands always appear near the pin. In the flashover experiment, partial arc first appeared near the pin, and then the cap of insulators, this phenomenon matches the dry bands distribution.

Before the appearance of the partial arc, the point-like corona discharges appear firstly, the direction of corona points is random, but the sheds are tend to occur, this is because that the water droplets are equal to the protrusion of insulator surface, it leads to the insulator surface field strength distribution change, the droplets come to the initial field strength of corona discharge at first. However, with the voltage increasing, partial arcs ignite the corona points will gradually disappear.

As a result of dry bands appearing, the voltage acts on the insulator string began to focus on dry bands, it results in breakdown of these regions, and then the partial arcs across the dry bands init. During the partial arc propagation process, the energy consumption increase, hence it may attenuate, even extinguish. As the humidification and drying are doubleaction process, dry bands may be re-humidity and narrow, which leads to the partial arc renewing. With the periodical changes of voltage and current, the brightness of partial arc will also periodically changes along with the leakage current, when the leakage current achieve maxim, the partial arc become brightest and when the leakage current passage zero, the partial arc become darkest. This phenomenon appears in both uniform and un-uniform contaminated situation.

When the lower surface of insulator contamination is heavy, upper surface contamination is light, after moist, the surface resistance of lower surface is greater than that of upper surface, by the action of the same leakage current, dry bands should appear on upper surface firstly, and then partial arc present. but it's not the fact. By observing the development process of partial arc, it is found that the initial partial arcs also appear near the pin of the insulator lower surface. Although the partial pressure of the upper surface is larger, the current density is not as concentrated as the foot of steel, once the dry band formed at lower surface, the field strength will be more concentrate on the dry bands compare to the upper surface and the situation of uniform contamination. Therefore, the initial partial arcs firstly appear at lower surface of insulator when the contamination of lower surface is much heavy than upper surface which is the same as uniform contaminated situation.

But in case of uniform surface pollution layer, the initial voltage decrease, the intense of partial arcs increase with the increase of ESDD value. Nevertheless, in case of un-uniform contaminated 
layer distribution, the initial voltage and intensive of partial arc not only relative with ESDD of the whole insulator strings, but the ratio of upper and lower surface ESDD. In case of the whole ESDD is constant, the initial voltage of partial arcs is minimal, the number is maximal, the development is soonest, and the discharge intensive is the most extreme when the ratio of upper and lower surface ESDD is 1:2. After that, the initial voltage increase, the propagation speed becomes slow with the decrease of the ratio, as a result, the critical arc gets shorter too.

The propagation modes of partial arcs are different in various pollution layer distributions:

In the circumstances of uniform pollution layer distribution, the primary partial arc propagation pattern is progressive-style, partial arc length changes with the voltage vary periodicity, but the length of latter period is always longer than earlier period. Fig. 2 is the partial arc gradually propagation process of $\mathrm{XWP}_{2}-160$ insulator string, the value of ESDD is $0.2 \mathrm{mg} / \mathrm{cm}^{2}$. (In this experiment, the speed of high-speed camera is 1000 picture per sec., hence the dwell time between two photos is $1 \mathrm{~ms}$ ). As can be seen in the lower figure, the partial arc becomes lighter, and then becomes dark, the lightness is almost in common with primary.

While ESDD value is $0.01,0.02$ or $0.05 \mathrm{mg} / \mathrm{cm}^{2}$, the partial arcs are lower brightness, slower propagation speed, higher igniting voltage. Relatively, while ESDD value is $0.1,0.2$ or $0.3 \mathrm{mg} / \mathrm{cm}^{2}$, the partial arcs are higher brightness, faster propagation speed and lower initial voltage.

References are cited in the text just by square brackets [1]. (If square brackets are not available, slashes may be used instead, e.g. /2/.) Two or more references at a time may be put in one set of brackets $[3,4]$. The references are to be numbered in the order in which they are cited in the text and are to be listed at the end of the contribution under a heading References, see our example below.

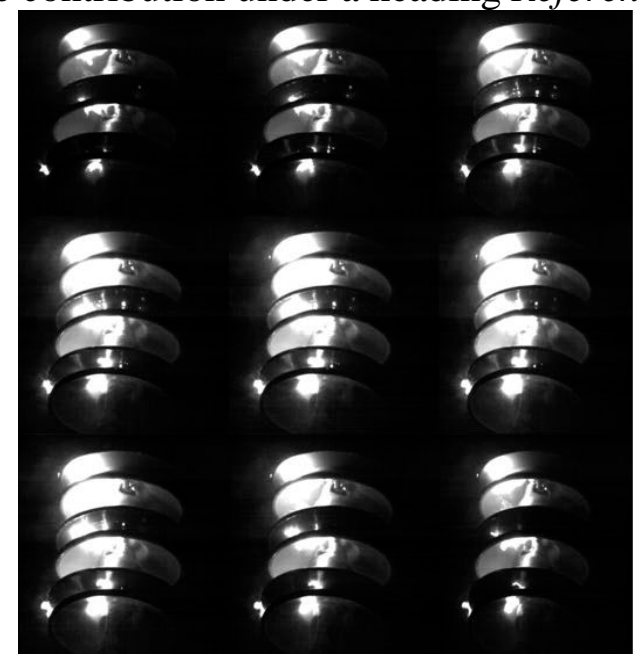

Fig. 2 The progressive-style propagation process

While the pollution severity of upper surface is less than lower surface, the partial arc propagation mode is the leap-style: thin discharge channel extend from the partial arc foot like tentacles. Then the thin discharge channels rapidly become as light as the original partial arc. The propagation process is shown in Fig. 3:

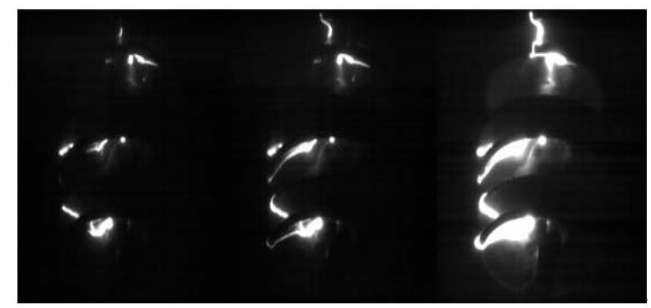

Fig. 3 The leap-style propagation process

In the case of uniform pollution layer distribution, the propagation mode of partial arcs is progressive-style, comparatively, in the case of un-uniform pollution layer distribution, the propagation mode of partial arcs is progressive-style and leap-style alternate.

During the propagation processes of partial arcs, partial arcs don't entirely close to the insulator surface, sometimes it short-circuit sheds of insulator string, that phenomenon is defined as arc-over, 
which is shown in Figure 4. Arc-over tend to occur while the ESDD value is bigger than $1 \mathrm{mg} / \mathrm{cm}^{2}$ in the case of uniform pollution layer distribution. While the surface contamination distributes un-uniformly, the probability of arc-over reduce. Arc-over shorten the leakage length of insulator strings, so that the withstand voltage drop.

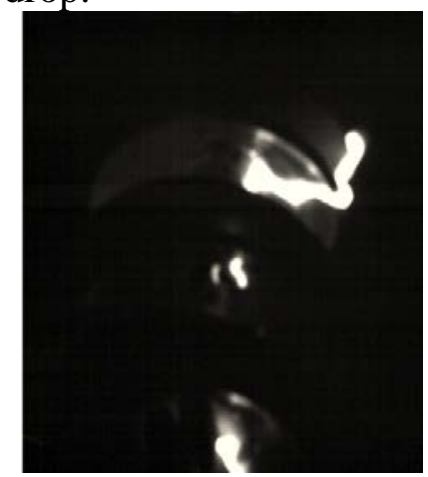

Fig. 4 Arc-over phenomena

\subsection{Analysis of Partial Arc Propagation Process.}

Before the flashover, the value of leakage current is small, at mA order of magnitude, only the ESDD value reaches to $0.2 \mathrm{mg} / \mathrm{cm}^{2}$, the leakage current value would exceed over $1 \mathrm{~A}$. At this time, partial arcs are darker, as shown in figure 5. At Pro-flashover time, the voltage drops rapidly from the peak, and the leakage current rise quickly. In the time of half period, it's value reach to dozens of amperes, the critical arc become lightening and throughout the whole insulator string rapidly.

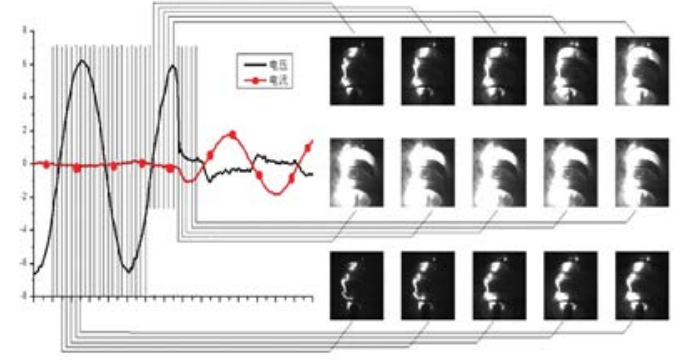

Fig 5 flashover process while ESDD value is $0.1 \mathrm{mg} / \mathrm{cm}^{2}$

In flashover process, there will be several current impulse at lower ESDD value (under $0.05 \mathrm{mg} / \mathrm{cm}^{2}$ ), the duration of impulse is about $40 \mu \mathrm{s}$, and the amplitude may be about $50 \sim 100 \mathrm{~A}$, the amplitude and the number of current pulse is irrelevant with ESDD, in the same value of ESDD, the number of flashover current pulse may be 1, 2, or 3, but no more than three. At same ESDD value, the amplitude of current pulse is lager at the situation of only one pulse than that of two or three pulses.

The current pulses in flashover process are shown in the two figures below this paragraph.

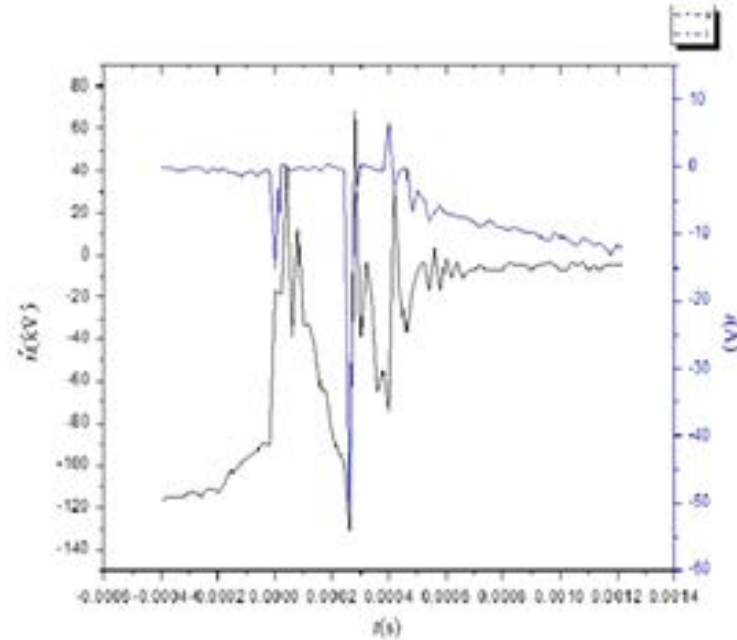

(a)

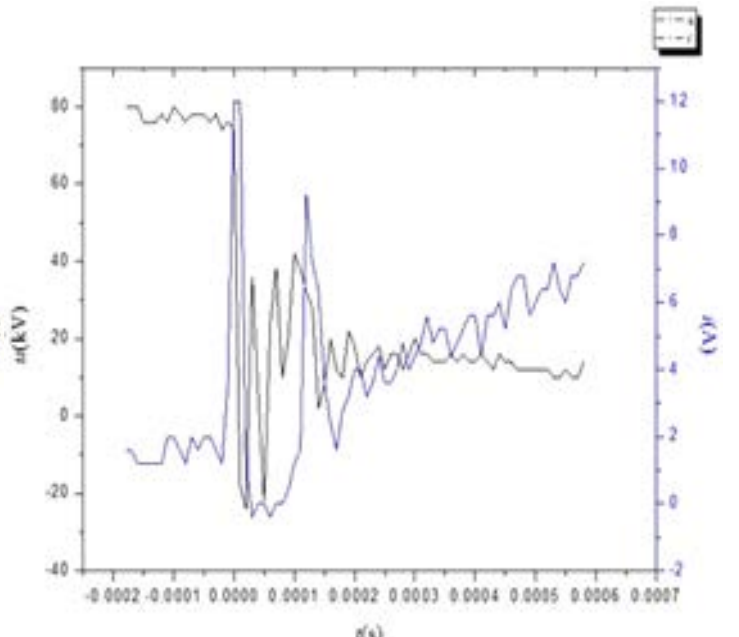

(b)

Fig. 6 flashover process while ESDD value is $0.01 \mathrm{mg} / \mathrm{cm}^{2}$ (a) or $0.05 \mathrm{mg} / \mathrm{cm}^{2}$ (b) 
The current pulses exist not only in flashover time but also all over the experimental process, and the pulse width is about $40 \mu \mathrm{s}$, they always appear at the leakage current zero-crossing time. It indicates that the current pulse phenomenon is not a coincidence. This may be due to the aerial discharge of partial arc propagation process. When partial arc get to critical flashover time, it's resistance reach to minimum value, a large share of voltage act on remain pollution layer, the temperature increase to the complete ionization point of $\mathrm{Na}^{+}$, result in intensive thermal ionization, the leakage current increases instantly. After the formation of plasma channel, the current reaches to a steady mode.

\section{Conclusion}

1) There are two partial propagation patterns, one is gradually-style, the other is leap-style. When the contamination distribution is uniform, the propagation mode of partial arcs is progressive-style, while at the situation of the pollution layer on upper surface is light and that of lower surface is heavy, the partial arc propagation pattern is alternation of leap-style and progressive-style.

2) Arc-over phenomenon appears in the case of uniform contamination, if the pollution layer distributes un-uniformly, the possibility of arc-over reduces.

3) In the case of un-uniform contamination distribution, the origination and propagation of partial arcs not only relate to ESDD of the whole insulator string, but the contamination distribution. When the ESDD ratio of upper and lower surface is $1: 2$, the partial discharge is the most intensive, and then alleviate with the ratio decreasing.

4) Un-uniform polluted deposition distribution decreases the possibility of arc-over.

5) The high frequency pulses of leakage current arise all over the whole energizing process, and the pulse width is constant as $40 \mu \mathrm{s}$, their position always nearby the zero-crossing of voltage waveform.

\section{References}

[1]. F. Obenaus, Fremdschicht "uberschlag und Kriechweglange, Deutsche Electrotechnik. 1958, 4: 135-136.

[2]. L.L. Alston, S. Zoledziowski, Growth of discharges on polluted insulation, Proc. IEE. 1963,110 (7): 1260-1266.

[3]. R. Wilkins, A. Al-Baghdadi, Arc propagation along and electrolyte surface, Proc. IEE. 1971, 118 (12) 1886-1892.

[4]. A.M. Rahal, C. Huraux, Flashover mechanism of high voltage insulators., IEEE Trans. Power Apparatus Syst. 1979, 98 (6): 2223-2231.

[5]. N. Dhahbi-Megriche, A. Beroual, Flashover dynamic model of polluted insulators under AC voltage, IEEE Trans. Dielect. Electr. Insul. 2000, 7 (2): 283-289.

[6]. Zhang Renyu, The development and prevention of electric discharge along a contaminated surface, Bulletin of National Science Foundation of China, 1996, 186-193 